\title{
Percepciones del entorno laboral de los profesores universitarios en un contexto de reorganización flexible del trabajo
}

Perceptions of the work environment of university professors within a context of flexible work reorganization

Perceptions du milieu de travail des professeurs universitaires dans un contexte de réorganisation flexible du travail

Francisco E. Restrepo Escobar

Profesor asistente, Politecnico Colombiano Jaime Isaza Cadavid,

Medellín - Colombia.

Magister en Gerencia del Talento Humano de la Universidad

de Manizales. Especialista en Alta Gerencia de la

Universidad María Cano y en Gestión del Talento Humano y la

Productividad de la Universidad de Medellín. Administrador de

Empresas de la Universidad de Antioquia.

E-mail: ferestrepo@elpoli.edu.co

Angélica M. López Velásquez

Profesora asistente, Universidad de Antioquia, Medellín - Colombia.

Magister en Gerencia del Talento Humano de la Universidad de Manizales. Especialista en Psicología Organizacional y Administradora de Empresas de la Universidad de Antioquia.

E-mail:amlopezv2@gmail.com

Artículo de investigación científica y tecnológica según clasificación COLCIENCIAS

Recepción: 06/03/2013

Corrección: 15/04/2013

Aprobación: 06/05/2013

\section{Resumen}

El propósito de la investigación consiste en analizar la subjetivación laboral sobre la calidad de vida laboral que los profesores universitarios construyen en torno a las políticas implementadas por las universidades en un contexto de reorganización flexible del trabajo. La metodología empleada contempla herramientas cuantitativas y técnicas cualitativas. Los resultados muestran que para la mayoría de los docentes hay un sesgo positivo frente a las condiciones de trabajo, el clima social, la satisfacción y la evolución laborales. Con respecto a los valores organizacionales, mostraron un sesgo positivo calidad del servicio, bien común, productividad, ética del servicio público y eficiencia, mientras que los que mostraron las medias más bajas y con un sesgo negativo fueron espíritu de negocio privado y rentabilidad económica.

Palabras clave: calidad de vida laboral, mercantilismo, managerialismo.

\section{Abstract}

The purpose of the research was to analyze labor subjectivation on the quality of working life faculty staff has built around the policies implemented by the universities within a context of flexible work reorganization. The methodology contemplated quantitative tools and qualitative techniques. The results show that while for most teachers a positive bias exists against working conditions, social climate, satisfaction and development, compared to organizational values, a positive bias was revealed regarding quality of service, common good, productivity, public service ethics, and efficiency. The lowest average with a negative bias and spirit was shown for private business and profitability.

Keywords: quality of working life, commercialism, managerialism.

\section{Résumée}

Le propos de la recherche est celui d’analyser la subjectivation au travail sur la qualité de vie au travail que les professeurs universitaires construisent autour de politiques implémentées par les universités dans un contexte de réorganisation flexible du travail. La méthodologie employée considère des outils quantitatifs et des techniques qualitatives. Les résultats montrent que pour la plupart des enseignants il existe une tendance positive face aux conditions de travail, le climat social, la satisfaction et l’évolution au travail. Quant aux valeurs organisationnels, ils présentent une tendance positive : la qualité du service, le bien commun, la productivité, l'éthique du service publique et l'efficacité. Les moyennes les plus basses et avec une tendance négative ont été : l'esprit d’entreprise privée et la rentabilité économique. 


\section{Introducción}

Los nuevos paradigmas de reorganización flexible del trabajo han permeado también a las instituciones de educación superior. "Las universidades ahora se reconocen como instituciones de mercado sujetas a la retórica sobre productividad, competitividad y rentabilidad en las que por ende comienzan a construirse nuevas percepciones sobre el entorno laboral" (Slaughter y Leslie, 2001) ${ }^{1}$.

En los últimos años la situación laboral en Colombia se ha caracterizado entre otros aspectos por mayores niveles de desempleo, el surgimiento de condiciones laborales precarias, inestabilidad laboral y sobrecarga ocupacional. Los trabajadores se hallan expuestos a presiones provenientes de su contexto laboral reaccionando ante ellas de diferentes formas, en función de ciertas características psicológicas y psicosociales. Cuando las presiones externas superan sus posibilidades de hacer frente a la situación, surgen factores psicosociales como el desinterés, la falta de concentración, la apatía, la disminución de la actividad, el estrés, etc. Bajo esta lógica, las universidades públicas van adquiriendo el rol de empresas del mercado caracterizadas por una reorganización flexible del trabajo. Así las cosas, nos concentraremos en las percepciones del entorno laboral que los profesores universitarios construyen en torno a las condiciones físicas y psicológicas del trabajo implementadas por las universidades para contribuir activamente a la generación de estrategias de autofinanciación en particular de la universidad pública. "Estas son presionadas para contribuir activamente en la competitividad de las economías locales-nacionales-regionales, estableciendo redes y circuitos de conocimiento con el Estado y las empresas, comercializando los productos de la investigación, generando estrategias de autofinanciamiento, asumiendo nuevos estilos de gestión y de marketing de corte empresarial, para ajustarse al nuevo escenario laboral flexible" (Gibb y Hannon, 2006). En este sentido, el capitalismo académico convierte lo que fue una "institución de la sociedad en una 'organización del mercado' (Ibarra, 2002), afectando la calidad de vida del personal docente.

Para entender lo anterior es preciso definir la calidad de vida como "un estado de satisfacción general, derivado de la realización de las potencialidades de la persona. Es una sensación subjetiva de bienestar físico, psicológico y social. Incluye como aspectos subjetivos la intimidad, la expresión emocional, la seguridad percibida, la productividad personal y la salud percibida. Como aspectos objetivos el bienestar material, las relaciones armónicas con el ambiente físico y social y con la comunidad, y la salud objetivamente percibida" (Ardila, 2003). En ese orden de ideas, y desde una perspectiva organizacional, la calidad de vida laboral (CVL) se puede lograr si se atienden los intereses organizacionales. Pero desde una perspectiva psicológica, la CVL se centra en el trabajador haciendo un microanálisis de los diferentes aspectos de las situaciones laborales en las que la persona participa directamente (González, 1996). Es esta pues una perspectiva más subjetiva.

De acuerdo con los conceptos enunciados, el presente estudio se centra en la dimensión subjetiva de la CVL, teniendo en cuenta las percepciones que los trabajadores docentes construyen sobre su entorno laboral; es decir, el acuerdo o desacuerdo con la actual organización del trabajo y las condiciones de trabajo (materiales, técnicas, financieras, contractuales, salariales, temporales, etc.) y la experiencia en relación con el clima social de trabajo. Las condiciones de trabajo son concebidas como el conjunto de las circunstancias y características ecológicas, materiales, técnicas, económicas, sociales, políticas, jurídicas y organizacionales en el marco de las cuales se desarrollan la actividad y las relaciones laborales. Surgen diversos criterios de clasificación de las condiciones laborales, que tienen que ver con el ambiente físico y social, de organización y las demandas cuantitativas y cualitativas, exigencias de rol, cargas y tiempos de trabajo que afectan la salud, la seguridad, al tipo y calidad del contrato laboral, y es lo que se denomina como bienestar laboral (Casales, 2003).

El bienestar laboral se puede estudiar desde cuatro enfoques: el enfoque ecologicista, que estudia y analiza la relación entre las personas y los espacios físicos que lo circundan. El enfoque económico del bienestar se centra en los niveles de ingreso, gasto social e inversión de los individuos, grupos o comunidades contemplados. La financiación y manejo de los recursos de una sociedad suele ser el rasero de medición vinculado con su grado de bienestar social. El enfoque sociológico se refiere al conjunto de condiciones objetivas y observables que conforman una comunidad. El grado de implementación de servicios y prestaciones de que disponga la comunidad, la influencia de variables demográficas, lugar de residencia o hábitat, junto con otras como el ocio, la familia o el trabajo, correlacionarán altamente con el bienestar general de las personas. Finalmente, el enfoque psicosocial contempla la interacción de la percepción de sí mismo y del mundo en el que se está inmerso. La construcción de recursos frente a las dificultades y conflictos de la realidad cotidiana será lo que se contempla en el bienestar psicosocial. Este concepto, relativamente joven y multidimensional, se acepta como integrador de otros como la felicidad o la satisfacción general con la vida. Se toman como criterios la intensidad y frecuencia de los estados afectivos. Se define operativamente la asociación de las emociones positivas y negativas vinculadas con sentimientos de confianza hacia el mundo que

1. Fenómeno que en la actualidad ha sido conceptualizado de diversas maneras: capitalismo académico (Slaughter y Leslie, 2001), capitalismo de los trabajadores del conocimiento (Bologna, 2006), managerialismo académico (Sisto, 2007), entre algunas otras. 
les rodea, la capacidad de manejo de los conflictos y la participación en actividades ocupacionales y laborales. La piedra angular de estas perspectivas es la satisfacción laboral, pues los resultados de la falta de satisfacción pueden afectar a la productividad de la organización y producir un deterioro en la calidad del entorno laboral. Puede disminuir el desempeño, incrementar el nivel de quejas, el ausentismo o el cambio de empleo. El bienestar laboral es el grado óptimo de satisfacción del trabajador, es un estado dinámico de la mente que se manifiesta conductualmente y que se caracteriza por la armonía entre las facultades, las necesidades y las expectativas del trabajador y las vigencias y oportunidades que el medio ambiente le brinda. El papel activo de la empresa moderna está formado por la inteligencia, comprensión, las habilidades y la experiencia de sus empleados. Una empresa logra su mayor ventaja cuando la cultura de los resultados y la estrategia se refuerzan mutuamente.

Por su parte, Slaughter y Leslie (1999) señalan que el capitalismo académico se refiere al uso que las universidades hacen de su único activo real, el capital humano de sus académicos, con el propósito de incrementar sus ingresos; tiene que ver con un conjunto de iniciativas y comportamientos económicamente motivados para asegurar la obtención de recursos externos. Esta tendencia nos permite poner en perspectiva nuevas prácticas de las universidades como la venta de productos y servicios con fines de autofinanciamiento, la competencia de los investigadores por financiamientos para sus proyectos - para acrecentar sus remuneraciones extraordinarias mediante programas de pago por mérito. Todo lo anterior llevó al equipo investigador a plantear el objetivo de la presente investigación, el cual consistió en analizar la subjetivación laboral sobre la CVL, específicamente las percepciones sobre el entorno laboral del personal docente de las universidades en un marco de reorganización flexible del trabajo.

\section{Metodología}

La investigación contempla un diseño mixto cuantitativo y cualitativo. La población objeto de estudio estuvo constituida por 141 profesores universitarios priorizándose los criterios de variedad, relevancia, significatividad y diversidad tipológica de contextos y situaciones organizacionales generadores de experiencias y discursos centrales en la subjetivación de la CVL. Por la misma razón, su tamaño no fue determinado por una fórmula estadística, sino en función del criterio de saturación informativa.

La significación de esta población no es estadística sino cultural, en términos de adecuación y suficiencia, es decir, los docentes participantes, por características como contar con un mínimo de dos años de pertenencia a la institución y ser ocasional o vinculado, pueden dar cuenta de las características de la cultura de la universi- dad pública objeto de estudio. Esto, por que interesa buscar el sentido que las personas confieren a un fenómeno.

Con respecto a la clasificación de la población, 52,8\% de los profesores tiene contrato estable y $44,4 \%$ inestable; $65,3 \%$ son hombres y $32,6 \%$ mujeres.

El estudio incluye el análisis de las siguientes variables: condiciones de trabajo, clima social de trabajo, satisfacción con la organización, evolución laboral y la influencia de los valores organizacionales en las políticas de la universidad, variables que influyen en el desgaste laboral (Blanch, 2010).

Las condiciones de trabajo incluyen el entorno físico, los recursos materiales y técnicos, los servicios auxiliares, el tiempo de trabajo, la organización general del trabajo, la carga de trabajo, la calidad del contrato, las oportunidades para la formación y la promoción laboral, la compatibilidad entre la vida laboral y la privada y familiar, así como la participación en las decisiones organizacionales.

La variable clima social de trabajo recogió percepciones frente al compañerismo, las relaciones con la dirección, el respeto en el grupo de trabajo, el apoyo recibido del personal directivo, el reconocimiento del propio trabajo por parte de colegas y de las personas usuarias del servicio, la evaluación del desempeño profesional, la autonomía en el ejercicio profesional y la justicia en la contratación, la remuneración y la promoción.

Satisfacción laboral incluye aspectos relacionados con la actual organización del trabajo como la satisfacción de intereses, la exigencia conforme a las capacidades, el encajamiento de dicha organización con las expectativas, el ajuste con las aspiraciones y valores, la valoración justa de los méritos, la motivación hacia el trabajo, la sensación de libertad, el desarrollo de las competencias profesionales y el estímulo del compromiso laboral.

En evolución laboral se evalúa entre otros aspectos la capacidad de gestión de la propia carga de trabajo, la autoestima profesional, la confianza en el futuro profesional, la sensación de seguridad en el trabajo, la realización profesional y el nivel de excelencia de la organización.

En la variable valores organizacionales se incluyeron valores mercantiles y sociales. Mercantiles como rentabilidad económica, calidad del servicio, competitividad, productividad, eficiencia y espíritu de negocio privado. Y sociales como bien común, calidad de vida laboral del empleado y ética de servicio público.

\subsection{Análisis de la información}

Los datos numéricos se procesaron mediante el programa estadístico SPSS, procediéndose desde el nivel descriptivo hacia tipos de análisis más complejos. En cuanto a los datos textuales, el trabajo fue orientado por el modelo de análisis cualitativo de contenido a través del Atlas Ti. Se realizó una triangulación de la información ob- 
tenida buscando los puntos de convergencia, de contraste y de complementación de sus respectivas aportaciones.

\section{Criterios de calidad de los reactivos de la encuesta}

\begin{tabular}{|l|l|l|}
\hline \multicolumn{2}{|c|}{ Tabla 1. Méto dos utilizados para evaluación del sistema } \\
\hline $\begin{array}{l}\text { Criterios de } \\
\text { calidad de los } \\
\text { reactivos }\end{array}$ & $\begin{array}{l}\text { Correlación } \\
\text { ítem-test }\end{array}$ & \multicolumn{1}{c|}{ Método } \\
\cline { 2 - 3 } & Confiabilidad & $\begin{array}{l}\text { Alfa de Crombach y dos } \\
\text { mitades de Guttman }\end{array}$ \\
\hline $\begin{array}{l}\text { Criterios de } \\
\text { calidad del } \\
\text { sistema }\end{array}$ & $\begin{array}{l}\text { Validez de } \\
\text { contenido }\end{array}$ & $\begin{array}{l}\text { Proceso adelantado por } \\
\text { expertos del proyecto } \\
\text { KOFARIPS2 }\end{array}$ \\
\cline { 2 - 3 } & $\begin{array}{l}\text { Validez de } \\
\text { construcción }\end{array}$ & $\begin{array}{l}\text { Método de extracción: } \\
\text { componentes principales }\end{array}$ \\
\hline Fuente: Restrepo, F.E.y López,A.M. (2013) basado en KOFARIPS (2010).
\end{tabular}

\section{Resultados y discusión}

\subsection{Análisis cuantitativo}

En relación con la variable condiciones de trabajo (Tabla 2), teniendo en cuenta que las respuestas tienen un sesgo positivo hacia las puntuaciones más altas, podemos concluir que los docentes de la universidad objeto de estudio, consideran que tienen buenas condiciones de trabajo.

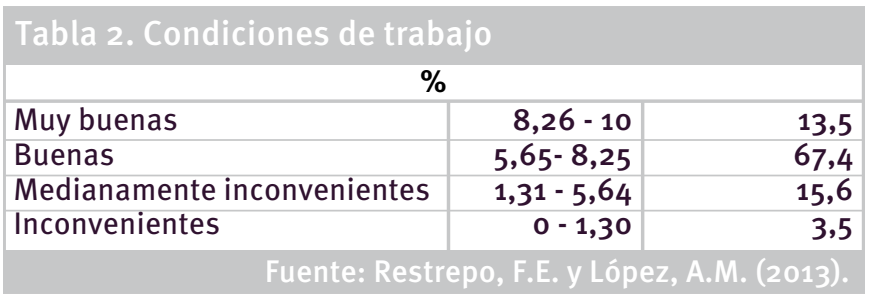

En relación con la variable clima social de trabajo (Tabla 3), puede inferirse que los docentes perciben que en la universidad objeto de estudio se cuenta con un buen clima social de trabajo.

Tabla 3. Clima social de trabajo
\begin{tabular}{|l|r|r|}
\hline \multicolumn{3}{|c|}{$\%$} \\
\hline Muy bueno & $\mathbf{8 , 8 2 - 1 0}$ & $\mathbf{1 5 , 6}$ \\
\hline Bueno & $5,95-8,81$ & 65,2 \\
\hline Medianamente inconveniente & $1,43-5,94$ & $\mathbf{1 4 , 9}$ \\
\hline Inconveniente Fuente: Restrepo, F.E. y López, A.M. (2013). \\
\hline
\end{tabular}

En conclusión y como lo indica la Tabla 4, los niveles de satisfacción de los docentes encuestados son altos (Tabla 4).

\begin{tabular}{|l|r|r|}
\hline \multicolumn{3}{|c|}{ Tabla 4. Satisfacción con la organización } \\
\hline Muy alta & $6,42-6,68$ & 17,0 \\
\hline Alta & $1,14-4,41$ & 68,1 \\
\hline Media & $1-1-13$ & 9,9 \\
\hline Baja & 5 \\
\hline
\end{tabular}

En relación con la variable evolución laboral (Tabla 5), más del $70 \%$ de la población docente encuestada considera que su evolución laboral es alta en relación con aspectos como motivación por el trabajo, calidad de las condiciones de trabajo, autoestima, conciliación trabajo familia, estado de ánimo, promoción laboral, seguridad en el trabajo, satisfacción con el trabajo, compromiso, competencias profesionales, entre otros.

\begin{tabular}{l} 
Tabla 5. Evolución laboral \\
\begin{tabular}{|l|r|r|}
\hline \multicolumn{3}{|c|}{$\%$} \\
\hline Muy alta & $6,42-7$ & 12,8 \\
\hline Alta & $4,40-6,41$ & 70,2 \\
\hline Media & $1,01-4,39$ & 12,8 \\
\hline Baja & $1-1-01$ & 4,3 \\
\hline
\end{tabular} \\
\hline
\end{tabular}

Se calcularon también los coeficientes de asimetría y curtosis confirmando la conclusión de que para el caso de las variables que acaban de analizarse, condiciones de trabajo, clima social de trabajo, satisfacción con la organización y evolución laboral, hay poca homogeneidad y simetría lo que corrobora el distanciamiento de la media y el sesgo positivo hacia las puntuaciones alta y muy alta (Tabla 6 ).

En relación con los valores calidad del servicio, bien común, competitividad, calidad de vida laboral del personal empleado, productividad, ética de servicio público y eficiencia, las desviaciones típicas indican que hay una baja dispersión de los datos. Es decir, el promedio en estos casos representa al grupo encuestado, viéndose así mayor homogeneidad en las respuestas de la muestra. Para los valores rentabilidad económica y espíritu de negocio privado, las desviaciones típicas indican que la dispersión de los datos es alta. Es decir, el promedio no representa a todo el grupo encuestado porque hay varios niveles (heterogeneidad) de percepciones en relación con los valores (Tabla 7).

\begin{tabular}{|c|c|c|c|c|c|c|}
\hline & \multicolumn{2}{|c|}{ Asimetría } & \multirow{2}{*}{ Cálculo As } & \multicolumn{2}{|c|}{ Curtosis } & \multirow{2}{*}{ Cálculo Curtosis } \\
\hline & Estadístico & Error típico & & Estadístico & Error típico & \\
\hline Condiciones de trabajo &,- 391 & ,204 & $-1,91636427$ &,- 226 &, 406 & $-0,55705077$ \\
\hline Clima social de trabajo &,- 651 & ,204 & $-3,18833696$ &,- 098 & ,406 & $-0,24135366$ \\
\hline Satisfacción con la organización & $-1,097$ & ,204 & $-5,37243277$ &, 811 & ,406 & 2,0 \\
\hline Evolución laboral &,- 825 & ,204 & $-4,04104004$ &, 262 & ,406 & 0,64518667 \\
\hline
\end{tabular}

2. Investigación internacional denominada “El capitalismo organizacional como factor de riesgo psicosocial”. Su interés particular es explorar y visibilizar las dimensiones relativas de las experiencias de trabajo del personal empleado en instituciones sanitarias y académicas reorganizadas bajo el nuevo modelo de gestión pública. Este estudio es liderado en Europa y Latinoamérica por Josep M. Blanch, docente del departamento de Psicología Social, de la Universidad Autónoma de Barcelona (España). 


\begin{tabular}{|l|r|r|r|r|r|}
\hline \multicolumn{5}{|c|}{ Estadísticos descriptivos } \\
\hline & N & Mínimo & Máximo & Media & $\begin{array}{c}\text { Desv. } \\
\text { típ. }\end{array}$ \\
\hline $\begin{array}{l}\text { Rentabilidad } \\
\text { económica }\end{array}$ & 140 & 0 & 10 & 6,02 & 2,26 \\
\hline $\begin{array}{l}\text { Calidad del } \\
\text { servicio }\end{array}$ & 140 & 2 & 10 & 7,28 & 1,69 \\
\hline $\begin{array}{l}\text { Bien común } \\
\text { Competitividad }\end{array}$ & 139 & 2 & 10 & 7,29 & 1,79 \\
\hline $\begin{array}{l}\text { Calidad de vida } \\
\text { laboral del } \\
\text { personal }\end{array}$ & 141 & 1 & 10 & 6,96 & 1,97 \\
\hline $\begin{array}{l}\text { Productividad } \\
\text { Ética de servicio }\end{array}$ & 141 & 2 & 10 & 7,28 & 1,61 \\
\hline $\begin{array}{l}\text { público } \\
\text { Eficiencia }\end{array}$ & 140 & 0 & 10 & 7,49 & 1,98 \\
\hline $\begin{array}{l}\text { Espíritu de } \\
\text { negocio privado }\end{array}$ & 136 & 2 & 10 & 7,03 & 1,72 \\
\hline & Fuente: Restrepo, F.E. y López, A.M. & & & \\
\hline
\end{tabular}

Los valores que en general mostraron un sesgo positivo (tendencia a la calificación más alta) fueron calidad del servicio, bien común, productividad, ética del servicio público y eficiencia. Mientras que los que mostraron las medias más bajas y con un sesgo negativo fueron espíritu de negocio privado y rentabilidad económica.

\begin{tabular}{|l|r|r|}
\hline \multicolumn{1}{|c|}{ Tabla 8. Cálculo del ADI (Average Deviation Index) } \\
\hline Rentabilidad & $\begin{array}{c}\text { Coeficiente de } \\
\text { dispersión }\end{array}$ & $\begin{array}{c}\text { Coeficiente de } \\
\text { variación }\end{array}$ \\
\hline Espíritu de negocio privado & 0,296 & 0,4 \\
\hline Calidad del servicio & 0,449 & 0,5 \\
\hline Bien común & 0,170 & 0,2 \\
\hline Competitividad & 0,175 & 0,2 \\
\hline Calidad de vida & 0,211 & 0,3 \\
\hline Productividad & 0,208 & 0,3 \\
\hline Ética de servicio & 0,152 & 0,2 \\
\hline Eficiencia & 0,184 & 0,3 \\
\hline
\end{tabular}

Se realizaron cálculos estadísticos complementarios como el ADM o ADI (Average Deviation Index) según los autores González-Romá et al., (2002) Los resultados, tal como se resume en la Tabla 8, confirman que para el caso de rentabilidad y espíritu de negocio privado, el coeficiente de variación es de $40 \%$ y $50 \%$ respectivamente (variabilidad alta) lo cual indica que el promedio no necesariamente representa a toda la población.

\subsection{Comparaciones entre grupos}

En relación con el tipo de contrato (Tabla 9), la prueba de Levene indica que para todos los casos, se han asumido varianzas iguales. La significatividad bilateral indica que las diferencias en las percepciones entre docentes ocasionales y vinculados no son significativas, con excepción de

\begin{tabular}{|c|c|c|c|c|c|c|c|}
\hline Variables & $\begin{array}{l}\text { Tipo de } \\
\text { contrato }\end{array}$ & $\mathbf{N}$ & Media & $\begin{array}{c}\text { Desviación } \\
\text { típ. }\end{array}$ & $\begin{array}{l}\text { Error típ. de } \\
\text { la media }\end{array}$ & $\mathbf{t}$ & $\begin{array}{c}\text { Sig. } \\
\text { (bilateral) }\end{array}$ \\
\hline \multirow[t]{2}{*}{ Condiciones de trabajo } & Estable & 76 & 7,2852 & 1,27766 & 0,14656 & 3,541 & 0,001 \\
\hline & Inestable & 64 & 6,5323 & 1,22341 & 0,15293 & 3,554 & 0,001 \\
\hline \multirow[t]{2}{*}{ Clima social de trabajo } & Estable & 76 & 7,4253 & 1,47823 & 0,16956 & 0,416 & 0,678 \\
\hline & Inestable & 64 & 7,3236 & 1,39514 & 0,17439 & 0,418 & 0,677 \\
\hline \multirow[t]{2}{*}{ Valores organizacionales } & Estable & 76 & 6,7054 & 1,29244 & 0,14825 & $-0,854$ & 0,395 \\
\hline & Inestable & 64 & 6,8906 & 1,26168 & 0,15771 & $-0,855$ & 0,394 \\
\hline \multirow[t]{2}{*}{ Satisfacción con la organización } & Estable & 76 & 5,5088 & 1,23877 & 0,1421 & $-0,45$ & 0,653 \\
\hline & Inestable & 64 & 5,5961 & 1,01668 & 0,12709 & $-0,458$ & 0,648 \\
\hline \multirow[t]{2}{*}{ Evolución laboral } & Estable & 76 & 5,4657 & 0,9963 & 0,11428 & 0,799 & 0,426 \\
\hline & Inestable & 64 & 5,3281 & 1,03704 & 0,12963 & 0,796 & 0,427 \\
\hline & & & & & \multicolumn{3}{|c|}{ Fuente: Restrepo, F.E. y López, A.M. (2013). } \\
\hline \multicolumn{8}{|l|}{ Tabla 10. Prueba T Sexo } \\
\hline Variables & Sexo & $\mathbf{N}$ & Media & $\begin{array}{c}\text { Desviación } \\
\text { típ. }\end{array}$ & $\begin{array}{l}\text { Error típ. de } \\
\text { la media }\end{array}$ & $\mathbf{t}$ & $\begin{array}{c}\text { Sig. } \\
\text { (bilateral) }\end{array}$ \\
\hline \multirow[t]{2}{*}{ Condiciones de trabajo } & Hombre & 94 & 7,1363 & 1,27367 & 0,13137 & 2,417 & 0,017 \\
\hline & Mujer & 47 & 6,5821 & 1,30319 & 0,19009 & 2,399 & 0,019 \\
\hline \multirow[t]{2}{*}{ Clima social de trabajo } & Hombre & 94 & 7,5046 & 1,44473 & 0,14901 & 1,379 & 0,17 \\
\hline & Mujer & 47 & 7,1522 & 1,40062 & 0,2043 & 1,394 & 0,167 \\
\hline \multirow[t]{2}{*}{ Valores organizacionales } & Hombre & 94 & 6,8916 & 1,19279 & 0,12303 & 1,313 & 0,191 \\
\hline & Mujer & 47 & 6,5937 & 1,41239 & 0,20602 & 1,242 & 0,218 \\
\hline \multirow[t]{2}{*}{ Satisfacción con la organización } & Hombre & 94 & 5,6499 & 1,10705 & 0,11418 & 1,402 & 0,163 \\
\hline & Mujer & 47 & 5,3658 & 1,18695 & 0,17313 & 1,37 & 0,174 \\
\hline \multirow[t]{2}{*}{ Evolución laboral } & Hombre & 94 & 5,4262 & 0,95131 & 0,09812 & 0,279 & 0,781 \\
\hline & Mujer & 47 & 5,3756 & 1,13678 & 0,16582 & 0,263 & 0,794 \\
\hline
\end{tabular}


la variable condiciones de trabajo frente a la cual las diferencias sí son significativas. Tiende a ser más positiva la percepción de los docentes vinculados.

En relación el sexo (Tabla 10) en el caso de la variable condiciones de trabajo, la significatividad bilateral indica que las diferencias en las percepciones entre hombres y mujeres son significativas. En las demás variables las diferencias no son significativas.

\subsection{Análisis cualitativo}

El análisis cualitativo si bien complementa el cuantitativo también introdujo categorías relevantes y nuevas como las siguientes: a1) lo inquietante del futuro laboral, a2) lo esperanzador del futuro laboral, b) la carga de trabajo y el tiempo para realizarla, c1) lo malo que va desapareciendo en el actual mundo de trabajo, $c_{2}$ ) lo bueno que se va perdiendo en el actual mundo de trabajo, d1) lo que va empeorando en el ambiente social de trabajo, d2) lo que va mejorando en el ambiente social de trabajo, e1) condiciones de trabajo que empeoran, e2) condiciones de trabajo que mejoran.

\subsection{Condiciones de trabajo que mejoran}

En relación con las condiciones de trabajo que mejoran, las categorías más recurrentes derivadas de las entrevistas apuntan a la disponibilidad de recursos tecnológicos y técnicos, percepción mencionada por la mitad de los profesores entrevistados. Plantean que se evidencia una mayor preocupación por parte de la administración por invertir en tecnologías de información y comunicación (TIC), que facilitan y cualifican el ejercicio de la docencia al tener, por ejemplo, acceso a información en tiempo real. Se incluye también el acceso a redes de información y de comunicación que le permiten al docente actualizarse en su área o áreas de interés. Muy asociada con la primera categoría es mencionada por las personas la adecuación del espacio físico señalando, especialmente, el mejoramiento de las condiciones de las oficinas en relación con las de períodos anteriores. Otra condición señalada por algunos docentes es la relativa a las condiciones contractuales, es decir, estos entrevistados consideran que tales condiciones han mejorado.

Otras condiciones que se señalan en proceso de mejoría, pero que no se han presentado de forma recurrente son el acceso a formación continua, el cambio en la concepción de lo público porque la universidad se ha venido moviendo de la lógica que homologa lo público con lo pobre o lo mínimo; la capacidad de gestión de algunas facultades con miras a su autosostenibilidad y a la creación de mejores condiciones para el trabajo; el clima laboral, lo que se aduce, influye positivamente en los resultados obtenidos; también se habla de una mejoría en el trabajo en equipo y finalmente para algunos de los entrevistados ninguna condición está mejorando.
Desde este punto de vista, la disponibilidad de recursos tecnológicos y técnicos, la adecuación del espacio físico y las condiciones contractuales se constituyen en condiciones claves a ser sostenidas porque contribuyen a elevar la calidad de vida laboral.

\subsection{Condiciones de trabajo que empeoran}

La cuarta parte de los docentes entrevistados expresa como condición que empeora la mercantilización de la universidad. El capitalismo académico constituye uno de los marcos de reflexión del presente estudio. Los docentes señalan que se pretende homologar la universidad con una empresa privada. Prueba de ello, la importación, sin analizar su pertinencia, de modelos económicos de producción que pretenden poner lo académico al servicio de lo administrativo y que obligan la celeridad en la producción para ser así eficientes, el aumento en la carga académica del profesor, la duplicación del número de estudiantes con los mismos recursos, desconociendo que los tiempos del conocimiento son otros; esta lectura de los docentes coincide con el planteamiento de Ibarra en cuanto a que la universidad está viviendo el proceso de dejar de ser una "institución" de la sociedad para pasar a ser tan sólo una "organización" del mercado (Slaughter y Leslie, 1999).

De la mano de la anterior percepción y también recurrentemente señalada, aparece la desmejora en la estabilidad laboral porque desde la óptica de los docentes ocasionales, cada vez se hace más evidente el hecho de que se está en la universidad de una manera temporal pues la posibilidad de vinculación no es clara ni fácil de lograr, lo que genera cierto desconcierto e intranquilidad y afecta obviamente la productividad y el desarrollo en el trabajo. Esta condición trae aparejados efectos personales porque planear o hacer proyecciones de vida a mediano o largo plazo se vuelve una utopía.

Además de las anteriores, se señalan otras condiciones del trabajo que han empeorado como la ausencia de bienestar laboral docente, la competencia desleal, la involución en la producción del conocimiento pues todavía la institución sigue anclada al paradigma de la información, la relación con compañeros, la salud mental y las relaciones de poder.

\subsection{Lo que va mejorando en el ambiente social de trabajo}

La categoría que más se destaca en este caso es la de relaciones interpersonales positivas. Se incluyen aquí las relaciones con colegas y jefes. Algunos docentes crean ambientes de solidaridad y compañerismo; un ejemplo muy claro que comparte uno de los docentes es el del trabajo gremial en el que "...hay una mayor colaboración, apoyo, discusión, debate, sin llegar a esos enfrentamientos durísimos... que se dieron en algunos momentos... Sí 
ha mejorado el ambiente de trabajo". Es importante recordar que así como la tarea común es clave para la cohesión de un grupo, también lo es el relacionamiento interpersonal positivo.

Otras categorías que emergen son el mejoramiento en el trabajo en equipo que posibilita la construcción colectiva y los canales de comunicación. Compañeros y jefes le prestan una mayor atención a los proyectos e iniciativas que se presentan. Se abren puertas y lazos laborales al interior y fuera de la universidad.

\subsection{Lo que va empeorando en el ambiente social de trabajo}

Al igual que en el caso de las condiciones de trabajo, la categoría que emerge de manera recurrente es la mercantilización de la universidad. Los docentes se refieren a una mayor solicitud de indicadores, a un énfasis en la productividad que hace que el sujeto pierda su rostro y su esencia. Todo esto por encima de la deliberación, de la crítica; con un número cada vez mayor de estudiantes y menos tiempo para acompañarles, la mayor presión de tiempo se orienta al cumplimiento de metas y no a la formación lo cual debería ser el centro. Pareciera que tal misión pasa a un segundo lugar; y por supuesto, las exigencias mencionadas, no dan cabida al encuentro con el otro.

Otra de las categorías recurrentes es la de relaciones interpersonales. Los docentes expresan que éstas se han vuelto distantes y poco transparentes. Lo primero, porque en la dinámica de trabajo cada uno se concentra en sus labores, aislándose de sus colegas. Y lo segundo, porque la competencia frenética y los beneficios aparejados a los logros individuales, hacen que las relaciones no sean transparentes, genuinas, auténticas y que se promueva el individualismo. Bajo ese panorama, ya no es posible ver al compañero como a un amigo "sino como alguien que te puede quitar el trabajo".

Son nombradas solo una vez otras condiciones que desmejoran en relación con el ambiente social de trabajo como el escaso tiempo para compartir con otros, la intolerancia a la crítica, la relación con los jefes, el menor compromiso organizacional y la escasa apertura al diálogo.

\subsection{Lo bueno que se va perdiendo en el actual mundo de trabajo}

El compañerismo y los espacios para compartir con colegas son las dos categorías que emergen con mayor recurrencia. El compañerismo entendido como solidaridad, cordialidad, amistad, camaradería.

Otra cosa buena que se va perdiendo y señalada por varios entrevistados es la disponibilidad de tiempo para el esparcimiento. Los docentes expresan que antes dedicaban más tiempo a espacios recreativos y a la práctica deportiva en la universidad y que todo ello ha sido relegado a un segundo plano por el trabajo; que incluso la familia se ha visto afectada por las demandas laborales.

Se hace mención a otras percepciones como la pérdida de las condiciones adecuadas para el ejercicio de la docencia por lo numeroso de los cursos, las presiones para la producción académica, la restricción a la libertad académica en cuanto a cómo y en qué proyectos invertir el tiempo. $Y$ se va perdiendo por la imposición de muchas decisiones demostrando que cada vez se tiene menos en cuenta la participación del profesorado en las decisiones institucionales, incluso, las que tienen que ver con su quehacer. Se señala también como ha desmejorado el equilibrio en la carga laboral lo cual restringe el tiempo para, como se anotaba, esparcirse y compartir con colegas.

\subsection{Lo malo que va desapareciendo en el actual mundo de trabajo}

Emerge una multiplicidad de categorías en relación con esta pregunta. Como ninguna recurre de forma significativa, se presentan a continuación por subgrupos. En el primero de ellos aparecen percepciones relacionadas con la actitud de apertura a la diferencia y a la participación como apatía a la participación, las diferencias culturales, los extremismos ideológicos y la ignorancia; en el segundo grupo aparecen asuntos relacionados con las relaciones con jefes, colegas y estudiantes que se considera son cosas malas que van desapareciendo como la distancia con directivos, la arbitrariedad en las decisiones, la invisibilidad profesional, la tensión en la relación con el estudiante y el poco compromiso del personal antiguo; en un tercer subgrupo aparecen las actitudes personales que pueden afectar la calidad del trabajo como la indisciplina, la ineficiencia y la mediocridad; el cuarto subgrupo reúne las percepciones de los docentes que se refieren a las condiciones laborales inadecuadas que van desapareciendo: la sobrecarga de trabajo, el trabajo bajo presión y el tiempo limitado para la administración académica. Quedan por fuera de estos subgrupos la metodología tradicional de enseñanza y las restricciones para la vinculación.

\subsection{La carga de trabajo y el tiempo para realizarla}

Solo un tercio de los docentes expresa que el tiempo para realizar su carga de trabajo le basta; uno de los docentes expresa que esto ocurre porque tiene una descarga académica que le permite no dictar clase y siendo la preparación de las clases una de las actividades más demandantes en términos de tiempo, ello lo libera. Para el grueso de los docentes falta tiempo por lo que se hace necesario trabajar más. Una de las razones que se aducen para esta situación es el plan de trabajo oculto, es decir, todas aquellas actividades informales, por fuera del plan de trabajo que son solicitadas al docente y que debe realizar, pero que no son tenidas en cuenta al momento de evaluar el desempeño; además de esta razón, la otra de más peso y que ha sido recurrente en 
muchas categorías indagadas es la sobrecarga laboral en relación con el tiempo disponible; los docentes expresan que en algunos casos la concertación de los planes de trabajo ha dejado de ser una concertación para convertirse en una imposición.

Los docentes que expresaron que les falta tiempo para llevar a cabo su carga de trabajo, plantean que la única forma de poder dar cumplimiento a los compromisos laborales es sacrificando espacios personales como compartir con la familia, con amigos o vivir momentos de esparcimiento, al llevar trabajo a casa. Esta situación genera intranquilidad, ansiedad y sensación de sometimiento.

\subsection{Lo esperanzador del futuro laboral}

Los entrevistados mencionan cerca de veinte percepciones diferentes en relación con lo que consideran esperanzador si se mira al futuro de su mundo de trabajo. Se destacan, porque son un poco más recurrentes, pero no de una manera muy significativa, una mayor cualificación y experiencia profesional y la vinculación a la universidad que posibilite la estabilidad laboral. Otras visualizaciones esperanzadoras se refieren a una distribución más equitativa de las cargas y el trabajo virtual que hará mucho más cómodo el trabajo del docente y le permitirá trabajar desde su casa.

\subsection{Lo inquietante del futuro laboral}

Las percepciones que más se destacan son la mercantilización de la educación universitaria, pues según la perspectiva de algunos docentes, se está centrando en producir técnicos carentes de civismo; en ese mismo orden de ideas, la privatización de la universidad también inquieta porque se llama la atención sobre el evidente interés del Estado en coaccionar las autonomías universitarias, los recortes disimulados de recursos que hacen pensar que no quieren sostenerla como universidad y que la dejan en un déficit presupuestal real.

De una manera general se solicitó a los profesores valorar el nivel de vida entre bueno, regular y malo obteniendo como resultado que $93 \%$ tienen un nivel de vida bueno. No se encontraron diferencias significativas en la calidad de vida, atendiendo las variables como el sexo y tipo de contrato.

\section{Conclusiones}

En esta investigación se ha estudiado la calidad de vida de los profesores de la universidad pública objeto de estudio desde una perspectiva subjetiva, a través de un diseño complejo que integra encuesta, entrevista y grupos focales.

Los estudios realizados al respecto revelan que las condiciones de trabajo inciden no sólo sobre la calidad del trabajo mismo, sino también sobre el bienestar, la salud, la motivación, el compromiso, la satisfacción y el rendimiento laborales, la eficacia y la eficiencia de las organizaciones y también sobre muchas de las disfunciones en las mismas, como conflictos, ausentismo y presentismo, rotación, accidentalidad o enfermedades laborales (Alcover et al., 2004; Peiró y Prieto, 1996). Esta se evaluó considerando lo que va mejorando o empeorando lo cual arrojó que para un $15.6 \%$ de los docentes las condiciones de trabajo son medianamente inconvenientes, mientras que $67,4 \%$ considera que son buenas y $13.5 \%$ de la población las considera muy buenas. Cabe anotar que las diferencias en las percepciones entre hombres y mujeres sobre las condiciones de trabajo son significativas. Tienden a ser menos negativas las percepciones de los hombres que de las mujeres. También son significativas las diferencias en las percepciones entre docentes ocasionales y vinculados en relación con la variable condiciones de trabajo. En este caso, tiende a ser más positiva la percepción de los docentes vinculados.

Los resultados de las entrevistas y grupos focales permiten entrar al detalle de esas condiciones de trabajo que son percibidas positivamente (que van en mejoría), pero también posibilitan conocer aquellas condiciones que desmejoran según los docentes. Puede concluirse que las principales condiciones del trabajo que mejoran son la disponibilidad de recursos tecnológicos y técnicos y la adecuación del espacio físico. Por el contrario, empeoran la mercantilización de la universidad, la cual se percibe como un aspecto inquietante del futuro laboral y la estabilidad laboral.

Con respecto a la variable clima social de trabajo referida al compañerismo, relaciones con la dirección, apoyo de los jefes y reconocimiento, los docentes consideran que es bueno en términos generales. Este resultado cuantitativo es confirmado por el análisis cualitativo porque las relaciones interpersonales positivas y el mejoramiento de las relaciones con los estudiantes son los factores que más se destacan en relación con lo que va mejorando en el ambiente social de trabajo. Sin embargo, llama la atención que también aparece recurrentemente la categoría de relaciones interpersonales como condición que desmejora por la distancia y poca transparencia de las mismas. Es decir, las relaciones interpersonales aparecen como factor psicosocial de riesgo y protector. También emerge de manera recurrente la mercantilización de la universidad como uno de los factores que empeoran en relación con el ambiente social del trabajo por la tendencia al individualismo producto del capitalismo académico.

Reforzando lo anterior, el compañerismo y los espacios para compartir con colegas y la disponibilidad de tiempo para el esparcimiento se destacan como lo bueno que se va perdiendo en el actual mundo de trabajo. En relación con los factores que dificultan estos actos de compañerismo se señalan la ausencia de espacios de discusión y las castas de profesores que perpetúan las diferencias.

Los resultados encontrados sugieren la revisión de los marcos legales como el estatuto profesoral cuyas más recientes reformas tienden hacia una mayor precarización de las condiciones de los docentes ocasionales. 
Otro de los planes que merece revisión y que de hecho ha generado recientes manifestaciones de protesta en la universidad objeto de esta investigación, es el de estímulos e incentivos. Si bien algunos de estos beneficios e incentivos cobijan a los docentes ocasionales, muchos otros los excluyen poniéndolos en una situación de desigualdad. Además, porque es un sistema que privilegia la producción investigativa frente al desempeño docente, atizando fenómenos como los que ya se han analizado ampliamente en este trabajo.

Cabe anotar que el análisis de grupo focal realizado permitió identificar cómo en relación con la salud física, la sobrecarga laboral comienza a generar dolencias físicas y mentales como la depresión. Algunos docentes afirmaron automedicarse y asistir enfermos a trabajar o continuar sus actividades laborales en casa a pesar de estar incapacitados.

Por último, se destaca el descontento docente respecto a la mercantilización de la universidad, por lo que se propone emprender estudios que debiliten la centralidad del management, tanto en la universidad en particular como en la sociedad contemporánea en general.

En un contexto laboral caracterizado por los sujetos como "burocrático", "rutinario", "flexible" y "mercantilista", las adecuadas condiciones de trabajo permiten el manejo de las emociones y emergen como un factor clave que contribuiría a prevenir el desgaste laboral.

La investigación aquí presentada es una contribución, no sólo al estudio de la dimensión subjetiva de la calidad de vida laboral, sino a la planificación de políticas educacionales y a la reflexión de cada profesor en relación con su actual mundo de trabajo y el futuro de la educación superior en general.

\section{Limitaciones}

Esta investigación es una primera fase exploratoria. Un segundo momento podría abarcar el modelo de cali- dad de vida laboral de la universidad pública objeto de estudio.

Se deben realizar investigaciones en el tema en otras poblaciones de docentes de diferentes universidades con el fin de obtener un perfil de calidad de vida laboral de profesionales que ejercen la labor docente.

\section{Referencias}

Anderson, G. (2008). Mapping academic resistance in the managerial university. Organization, 15 (2) (pp. 251-270).

Alcover, C.M., Martínez, D., Rodríguez, F. y Domínguez, R. (2004). Introducción a la Psicología del Trabajo. Madrid, España: McGraw-Hill.

Ardila, R. (2003). Calidad de vida: Una definición integradora. Revista Latinoamericana de Psicología, 35 (2) (pp. 161-164).

Blanch, J.M., Sahagún, M., Cantera, L. \& Cervantes, G. (2010). Questionnaire of general labor well-being: structure and psychometric properties. Journal of Work and Organizational Psychology, 26 (2) (pp. 157-170).

Casales, J. (2004) Psicología Social. La Habana, Cuba: Editorial Félix Varela.

Casas, J., Repullo, J., Lorenzo, S. y Cañas, J. (2002). Dimensiones y medición de la calidad de vida laboral en profesionales sanitarios. Revista Administración Sanitaria, VI (23) (pp. 144-160).

Gibb, A.A. \& Hannon, P.D. (2006). Towards the Entrepreneurial University? International Journal of Entrepreneurship Education, 4 (1) (73-110).

González, P. y Bousoño, M. (1996) Evaluación de calidad de vida. Psiquiatría, V (6) (pp. 47-54).

González-Romá, V., Peiró, J.M. \& Tordera, N. (2002). An examination of the antecedents and moderator influences of climate strength. Journal of applied psychology, 87 (3) (pp 465-473).

Ibarra, E. (2002). La nueva Universidad en México: transformaciones recientes y perspectivas. Revista Mexicana de investigación educativa, 7 (14) (pp. 75-105).

Peiró, J.M. y Prieto, F. (1996).Tratado de psicología del trabajo II. Cap. 3. Madrid, España: Ed. Síntesis.

Slaughter, S. \& Leslie, L. (1997). Academic capitalism: politics, policies, and the entrepreneurial university. Baltimore MD, USA: Johns Hopkins University Press (p. 296).

Cuadernos de Administración / Facultad de Ciencias de la Administración / Universidad del Valle Periodicidad: semestral / ISSN impreso Nº120-4645-ISSN electrónico N² 2256-5078 / Nombre abreviado: cuad.adm. Edición Vol. $29 \mathrm{~N}^{\circ} 49$ (enero - junio de 2013)

Percepciones del entorno laboral de los profesores universitarios en un contexto de reorganización flexible del trabajo/ Francisco E. Restrepo Escobar y Angélica M. López Velásquez 